\title{
Atmospheric Light Estimation using Particle Swarm Optimization for Dehazing
}

\author{
Padmini. T. ${ }^{1}$ \\ Department of Embedded Technology \\ School of Electronics Engineering \\ Vellore Institute of Technology (VIT) \\ Vellore, India
}

\author{
Shankar. $\mathrm{T}^{2}$ \\ Department of Communication Engineering \\ School of Electronics Engineering \\ Vellore Institute of Technology (VIT) \\ Vellore, India
}

\begin{abstract}
For the past decade, many researchers have been working towards the improvement in the visibility of single hazy images, using the haze image model. According to the haze image model, the hazy-free image is restored by estimating the atmospheric light and transmission from a hazy image. The objective of this proposed work is to improve the perceptibility by decreasing the density of haze in the hazy images. The research work was carried to estimate the optimal value of atmospheric light by tuning the weights using a bioinspired technique called Particle Swarm Optimization (PSO) based on the objective of minimizing the fog density. We have selected a fitness function or objective function which incorporates all statistical features to differentiate a clear image from the hazy image. The results are validated with the state-of-the-art, by measuring fog density of the restored image using Fog Aware Density Evaluator (FADE). Also, the results are validated by measuring the Peak signal to noise ratio (PSNR) and structural similarity index (SSI) using ground truth images from Foggy Road image database (FRIDA). This research work demonstrates better results qualitatively and quantitatively.
\end{abstract}

Keywords-Hazy images; particle swarm optimization; dark channel prior; transmission; atmospheric light

\section{INTRODUCTION}

Fog, haze, smoke, rain, and snow are the factors which deteriorate the perceptibility of outdoor images. The conditions of the weather vary primarily in size, type, and concentration of the particles in the space. Opaqueness is formed in the lower atmosphere, due to fine suspended particles. Haze affects the perceptibility because it produces a unique gray hue [1]. The difference between haze and fog is that the particles of haze are smaller than fog droplets but slightly larger than air molecules. Similarly, the difference between cloud and fog is that the former exists at a higher altitude than the latter which is at the ground level. As we hinder ourselves to ground level vision, clouds are of less pertinence to us [1].

Fig. 1 shows the effects of scattering of light due to suspended particles in the atmospheric. According to Koschmieder, the light from the sun falls on the scene and gets reflected back to the camera is known as direct transmission. But due to suspended particle like haze, fog and smoke in the atmosphere the light gets scattered in different directions and some portion of the light reaches the camera is known as airlight. Dehazing algorithms were developed based on enhancement and restoration based methods. Restoration based methods use the haze image model which is expressed in equation (1) as follows:

$$
H(x, y)=f(x, y) t(x, y)+A(1-t(x, y))
$$

where $H(x, y)$ is the hazy image, $f(x, y)$ is the clear image, $A$ is the atmospheric or ambient light and $t(x, y)$ is transmission which is expressed in equation (2) as follows,

$$
t(x, y)=e^{-b d(x, y)}
$$

Transmission refers to the light that is reaching the camera from the scene and its value varies between 0 and 1 . The exponentially decaying light depends on depth $d(x, y)$ and scattering coefficient $b$.

Thus from the Koschmieder equation (1), we can conclude that the hazy image has low contrast by the attenuation term $\mathrm{f}(\mathrm{x}, \mathrm{y}) \mathrm{t}(\mathrm{x}, \mathrm{y})$ and whiteness is blended with the image because of the airlight term $\mathrm{A}(1-\mathrm{t}(\mathrm{x}, \mathrm{y}))$.To obtain the haze-free image, contrast of the image should be improved and colour correction has to be done [2]. Most of the researchers use restoration methods to restore the haze-free $(\mathrm{f}(\mathrm{x}, \mathrm{y}))$ parameter using the haze image model by calculating the transmission ( $\mathrm{t}$ ) and atmospheric light (A).

In Section 2, the survey of past work in haze removal is discussed. Section 3 explains our proposed work of dehazing using PSO, Section 4 describes outcome of our work. Finally, Section 5 ends with a conclusion.

\section{RELATED WORK}

The colour and contrast of the images get deteriorated drastically under inclement weather conditions. With the increase in distance between the camera and the object, the level of degradation also increases. Image de-hazing problem was solved by using multi-image technique initially. When only a single deteriorated image is available, then the task becomes more challenging. There have been several algorithms proposed in the past few years for removal of fog using a single image. Information regarding the depth is estimated in these algorithms and it can be determined by categorizing the algorithm into two: interactive or automatic restoration technique. 


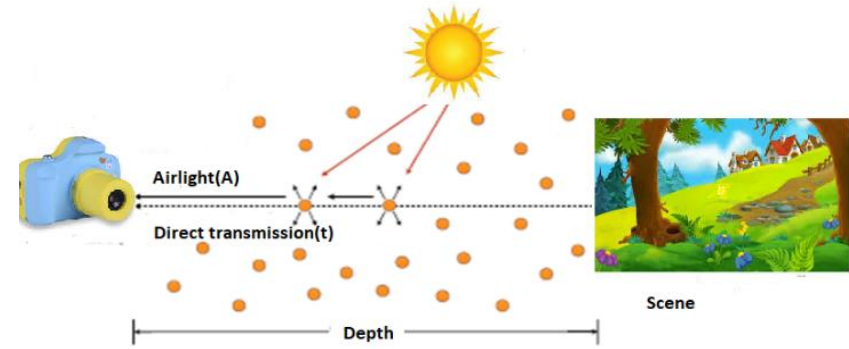

Fig. 1. Pictorial Representation of Effects of Atmospheric Scattering.

Based on the 3D model of the scene, Kopf [3] proposed a procedure applying which can remove the haze effects easily if the information about the depth is known at each pixel. His work is based on interactive restoration technique. Under the automatic restoration technique, there are several methods which have been proposed to remove the haze without the intervention of the user using the single hazy input image. Oakley and $\mathrm{Bu}$ [4] developed a model which gives a way to perceive the presence of airlight. It is assumed that the airlight throughout the image is constant. Contrast loss can be corrected easily once the level of airlight is determined. This algorithm falls short when there is no uniformity in airlight over the image. Tripathi [5] has discussed about the interactive and automatic methods in his review paper. Kim [6] improvised the method recommended by Oakley and $\mathrm{Bu}$ [4] so that it can be applied even when the airlight is variable over the image. The airlight is estimated by using the luminance image which is determined by the combination of R, G, and B colour component. The airlight map generated is used for restoring the image by getting subtracted from the foggy image. Region segmentation is performed for estimating the airlight in each region which is reflected by depth variation. This method falls short in covering a wide range of depth of the scene. Tan [7] proposed a method based on spatial regularization from the grayscale or single colour image. The image restored produces halos and looks saturated. Independent Component Analysis (ICA) is the concept which Fattal [8] used in his work. To estimate the transmission, Fattal considered ICA. Optical transmission is estimated in the hazy scene and based on this, to increase the scene perceptibility, the scattered light is eliminated. This technique cannot be used for a grayscale image as the colour information is required for restoration. Dense fog is colourless and hence this technique falls short when the fog is very dense. He [9] proposed an effective method involving soft matting and dark channel prior. It is an innovative approach adopted by most researchers which explain as follows, the natural clear images except sky region was found to have pixels with shades of black in any one of the R, G, and B channel. The algorithm uses Levin's soft matting way to improve the transmission map which is computationally expensive. Tarel and Hautiere [10] introduced a computationally effective algorithm known as fast visibility restoration in which airlight is expected to be the percentage between the local average and deviation from the average of the whiteness. The contrast is maximized for the resulting image and it is assumed to have a smooth depth map all over except along the edges. In the case of discontinuities in depth, the quality of the restored image is not so good. Zhu [11] proposed a method based on colour attenuation prior, where the estimation of the depth depends on the difference between brightness and saturation. Once depth is estimated transmission can be determined. But this method fails at edges. Dark channel prior fails to work at sky region. To overcome this problem Wang [12] and Li [13] segmented the sky and other regions separately from outdoor images to estimate and remove the haze from images. Recently, researchers are focusing on implementing optimization algorithms to improve the perception of hazy images based on haze image model. Guo [14] in his work as tuned the aerial perspective and lower bound of transmission for recovering scene radiance using Genetic algorithm. Zhang [15] used Ant colony optimization algorithm using k means clustering to evaluate atmospheric light. Optimization algorithms outperform other methods by obtaining visually compelling results. Recently, Singh [16] has reviewed dehazing algorithm explaining various techniques adopted by researchers in this field.

\section{Proposed Method for De-HAZING}

A simple and efficient method is implemented by extending the work of Dark channel prior by tuning the parameters suitably using Swarm intelligence optimization technique, which is an exhaustive search method for obtaining an optimal value for atmospheric light (A). Also, while calculating the transmission, the aerial perspective $\omega$ and while restoring the haze-free image the lower bound of transmission t0 are suitably tuned as specified by Guo [14], using Particle swarm optimization PSO [17] so that the hazefree image is visually compelling. The step-by-step framework for dehazing is summarized in Fig. 2.

For the restoration process, hazy colour image $H^{c}(x, y)$ is required as the input. To obtain an optimal value for atmospheric light, initially dark channel is obtained from the input hazy image. As per He [9], the dark channel $H^{\text {dark }}(x, y)$ is given by,

$$
\left.H^{\text {dark }}(x, y)=\min _{\Omega(x)}\left(\min _{C \in(R, G, B)} H^{C}(x, y)\right)\right)
$$

where $\Omega(\mathrm{x})$ is a local square window, $\min$ is the minimum filter, $H^{c}(x, y)$ refers to colour channel R, G, B of the input image $H(x, y)$. Dark channel inclines towards zero for clear image. So the equation (3) can be written as

$$
H^{\text {dark }}(x, y) \rightarrow 0
$$

For optimizing the atmospheric light $A$, we followed the weighted method proposed by Shiau [18]. According to Shiau, the atmospheric light is related to illumination of the restored images. If a hazy image as both dark and bright regions then computing the brightest pixel alone from the dark channel will not increase the visibility of the output image. In the weighted method, the threshold value $\mathrm{T}$ is obtained from the grayscale of the input hazy image which is used to divide the dark channel regions into bright and dark regions. We took the patch size as $3 \times 3$ for computing dark channel as per Shiau [18]. The pixel values higher than $\mathrm{T}$ will be under bright region and the other pixel are under the dark region. 


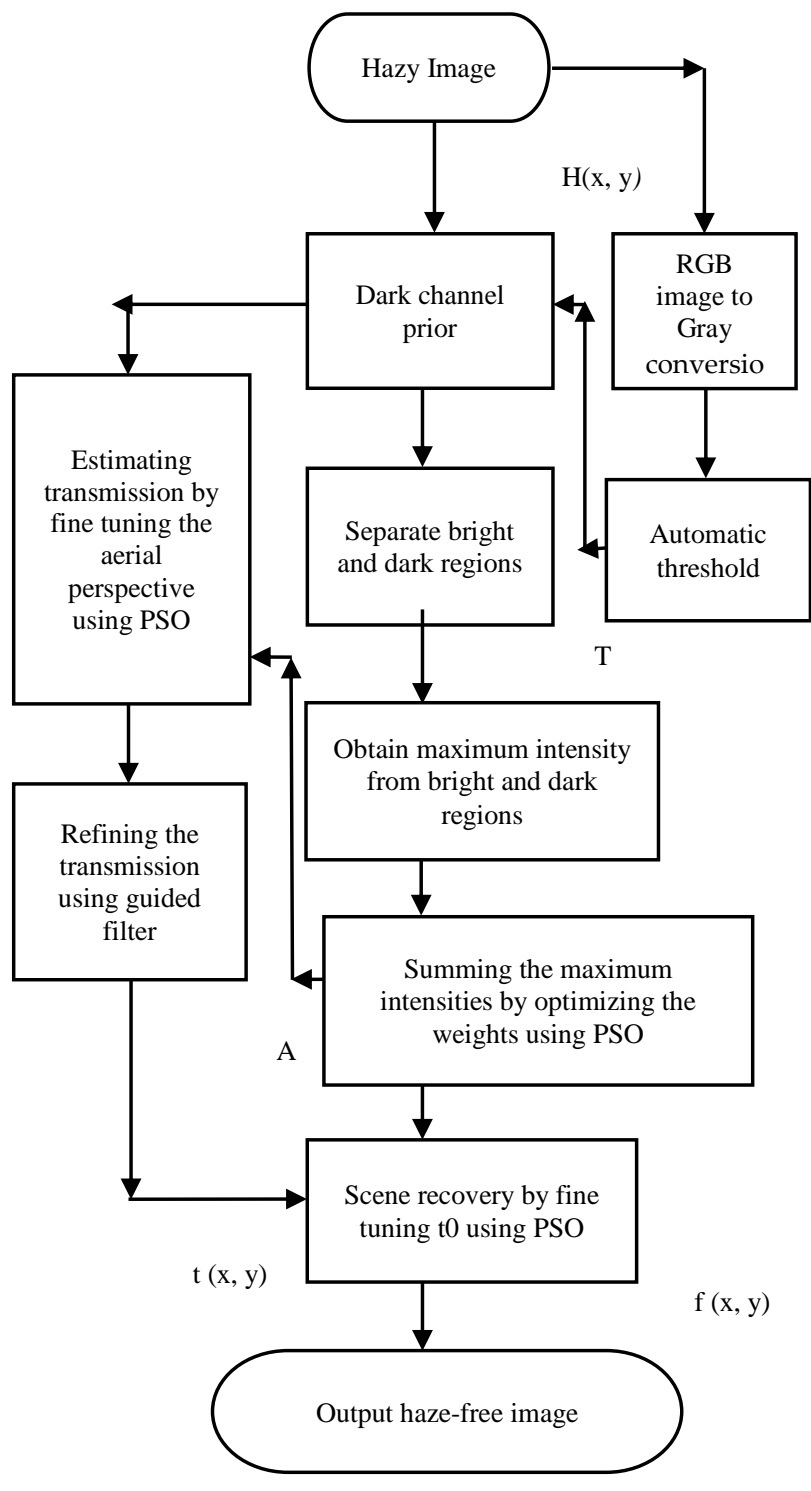

Fig. 2. Overview of our Proposed Method for Dehazing.

The maximum intensities in both the dark and bright regions are found out to compute the atmospheric light. This can be calculated as follows:

$$
\begin{aligned}
& A_{\text {brightf }}=\max _{(x, y) \in \text { bright }}\left\{H^{\text {dark }}(x, y)\right\} \\
& A_{\text {darkf }}=\max _{(x, y) \in \text { dark }}\left\{H^{\text {dark }}(x, y)\right\}
\end{aligned}
$$

where $H^{\text {dark }}(x, y)$ is the dark channel of the input hazy image. Let $\left(\mathrm{s}_{1}, \mathrm{t}_{1}\right)$ and $\left(\mathrm{s}_{2}, \mathrm{t}_{2}\right)$ are the coordinates where $A_{\text {brightf }}$ and $A_{\text {darkf }}$ are located. To obtain the atmospheric light, the corresponding coordinate pixels of the above said parameters in the input haze image are taken and is expressed as:

$$
A=W_{b} * H^{C}\left(s_{1}, t_{1}\right)+W_{d} * H^{C}\left(s_{2}, t_{2}\right)
$$

where $H^{c}\left(s_{1}, t_{1}\right)$ and $H^{c}\left(s_{2}, t_{2}\right)$ are the corresponding pixels of $A_{\text {brightf }}$ and $A_{\text {darkf }}$ respectively in the input hazy image $H(x, y), W_{b}$ and $W_{d}$ are the weights assigned to brighter and dark regions respectively. ' $*$ ' indicates the multiplication symbol. The total weight,

$$
W_{b}+W_{d}=1
$$

In our proposed method, the weights $W_{d}$ and $W_{b}$ are optimized using PSO [17] satisfying the equation (8). Using the optimized weight, atmospheric light is estimated. Shiau [18] in his work used a fixed weighted value by considering the sum of the probability within dark region and within bright region.

Before implementing our research work, we tested using different fixed values of weights for calculating the atmospheric light without using optimization technique. Fig. 3 shows the restored image and the effect of atmospheric light when the weights are varied with different fixed values retaining the other parameters used for calculating atmospheric light by Shiau [18].

From the visual comparison of the images in Fig. 3, the significance of weights of atmospheric light can be determined. Hence an optimal weight will solve this problem by improving the quality of the image. Transmission can be obtained once an optimal value of atmospheric light is determined.

The transmission is estimated as per He's [9] approach as follows:

$$
t=1-\omega \min _{\Omega(x)}\left(\min _{C \in R, G, B}\left(\frac{H(x, y)}{A}\right)\right)
$$

where $\omega$ is a constant known as aerial perspective whose value lies in the range $0<\omega \leq 1$. Generally, $\omega$ is fixed as a small value to maintain naturalness of the scene. Based on He's [9] observation, the dark channel $H^{\text {dark }}(x, y)$ is very close to zero, the equation (9) was obtained which is as per the definition of the dark channel prior. In our proposed method the aerial perspective $\omega$ is chosen as an optimization parameter to fine tune to get an optimal solution. Since the transmission is assumed to be constant within the window, halo artifacts might occur and so for eliminating the halos, we use edge-preserving smoothing guided filter [19] to refine the transmission. Hence, the restored clear image $f(x, y)$ can be expressed as. 


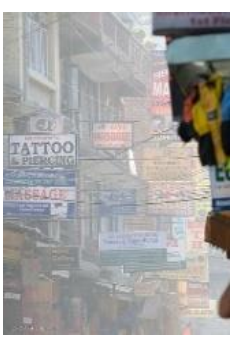

(a)

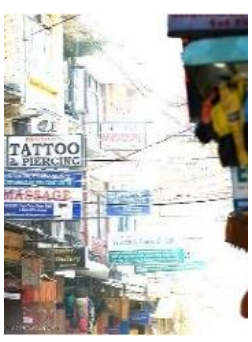

(b)



(c)

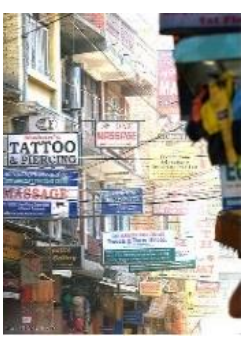

(d)

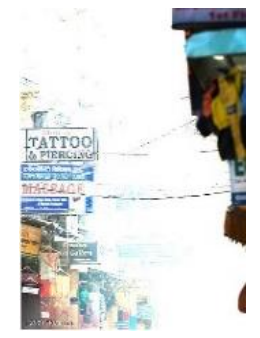

(e)

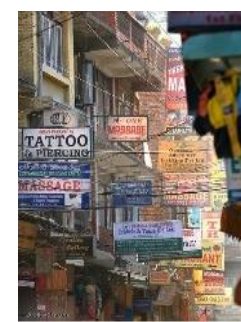

(f)

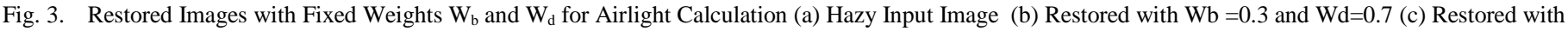
$\mathrm{Wb}=0.7$ and $\mathrm{Wd}=0.3$ (d) Restored with $\mathrm{Wb}=0.5$ and $\mathrm{Wd}=0.5$ (e) Restored with $\mathrm{Wb}=0.1$ and $\mathrm{Wd}=0.9$ (f) $\mathrm{Restored}$ with $\mathrm{Wb}=0.9$ and $\mathrm{Wd}=0.1$.

$f(x, y)=\frac{(H(x, y)-A)}{\max \left(t(x, y), t_{0}\right)}+A$

where $t 0$ refers to the lower bound of the transmission which is optimized using PSO to get a better quality of scene radiance at the end of the restoration process as discussed by Guo [14] who used genetic algorithm to tune the parameters.

\section{A. Implementation of PSO for Dehazing}

In this section, we propose the implementation of PSO for dehazing choosing an optimal weight $\left(W_{b}, W_{d}\right)$ for estimating atmospheric light (A) as specified in equation (7), the optimal value for aerial perspective $(\omega)$ as specified in equation (9) and optimal value for lower bound of the transmission $\left(t_{0}\right)$ as specified in equation (10). Fig. 4 represents a complete framework of the implementation of an optimization technique to obtain the haze-free image.

In this research work, restoration is considered as an optimization problem and the details are summarized. The conventional PSO algorithm is an exhaustive search based algorithm which considers a collection of particles as Swarm. Let us consider there are $\mathrm{N}$ particles in the search space. The optimizing parameter can be represented as follows:

Weights for estimating the atmospheric light $W_{d}=\left(W_{d l}\right.$, $\left.W_{d 2}, \ldots \ldots \ldots, W_{d N}\right)$ and $W_{b}=\left(W_{b 1}, W_{b 2}, \ldots \ldots \ldots, W_{b N}\right)$. Similarly, aerial perspective $\omega=\left(\omega_{1}, \omega_{2}, \ldots \ldots \ldots \ldots \ldots \omega_{N}\right)$ and lower bound of the transmission $t_{0}=\left(t_{01}\right.$, $\left.t_{02}, \ldots \ldots \ldots \ldots \ldots \ldots \ldots t_{0 N}\right)$.

For each iteration, and for each particle, the optimizing parameters $\left(W_{d}, W_{b}, \omega, t_{0}\right)$ are used to perform the restoration process of the hazy image using the equation (7), (8), (9) and (10). After restoration, the images are evaluated using fitness function.

Since the objective is to minimize the fog density of the restored image, the fitness function or objective function is appropriately chosen in our proposed method to calculate the fog density ' $\mathrm{D}$ ' of the restored image, referred as Fog aware density evaluator (FADE) proposed by Choi [20]. By fine tuning, the parameters like weights in estimating the atmospheric light, aerial perspective and lower bound of the transmission, the fitness function is minimized to obtain an optimal solution using Particle Swarm Optimization.

FADE computes the density of fog in an image, proposed by Choi [20], by considering the statistical features of 500 hazy and 500 clear images. The statistical features used to compute fog density is explained as follows.

Mean Subtracted Contrast Normalized (MSCN) coefficients obtained from outdoor clear scenes and from foggy images are computed as follows:

$$
I(M S C N)=\frac{I_{\text {gray }}(i, j)-\mu(i, j)}{\sigma(i, j)+1}
$$

where $I_{\text {gray }}$ is the gray scale of the input image, $\mu$ is the mean and $\sigma$ is the standard deviation. As per Choi [20], Normalized Histogram was plotted using MSCN coefficients and found that for denser foggy images histogram incline away from Gaussian whereas the MSCN coefficient of clear images is very close to unit normal Gaussian. The variance of the MSCN coefficient is considered as one of the features. Similarly, the variance of the positive and negative mode of the vertical product of MSCN coefficients is considered as another feature for computing fog density. Apart from MSCN coefficients for measuring fog density, Choi [20] used other statistical features like sharpness which is described by the local standard deviation $\sigma(\mathrm{i}, \mathrm{j})$, the coefficient of variation of sharpness, Contrast energy obtained separately from gray, yellow-blue and red-green channel of the Image. Entropy is another feature used since very fewer details are contained in foggy images. The novel feature is the pixel-wise dark channel prior introduced by $\mathrm{He}[9]$ is used. It is found using the equation (3) discussed in dark channel prior topic. As the foggy region is affected by colour, to measure its perceptibility, the fog aware feature used is colourfulness and colour saturation. The degree of difference between gray and colour is colourfulness and the amount of whiteness blended with colour is saturation. With an increase in fog density on the image, the colourfulness and saturation decrease. This is because of the colour shift caused due to airlight scattering. Colourfulness is calculated from the standard deviation and mean of red-green and yellow-blue plane using the following expression: 
Hazy Image

Haze free Image

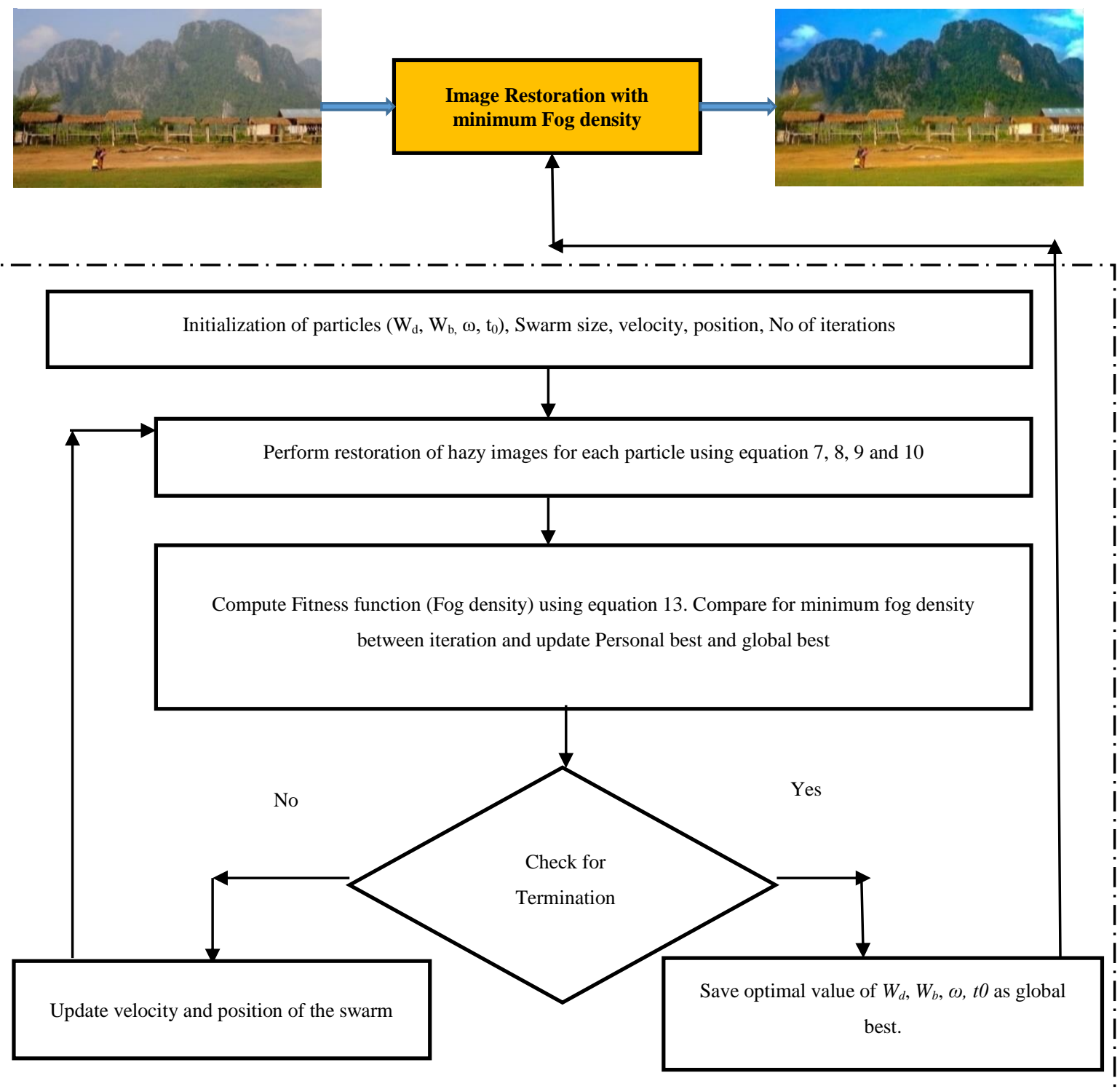

Fig. 4. Framework for Dehazing based on PSO.

$$
C F=\sqrt{\sigma_{r}^{2}+\sigma_{y}^{2}}+0.3 \sqrt{\mu_{r}^{2}+\mu_{y}^{2}}
$$

where $\sigma_{r}, \sigma_{y}, \mu_{r}, \mu_{y}$ are standard deviation and mean of red-green and yellow-blue plane respectively.These statistical features are used to fit as a Multivariate Gaussian model (MVG) to compute the fog density. Fitness function in our approach is chosen which considers all the statistical features used to differentiate between clear and hazy image is specified in equation (13),

$$
D=\frac{D_{f f t}}{D_{f t}+1}
$$

where $D_{f f t}$ is the Mahalanobis distance between MVG models obtained using statistical features acquired from 500 clear image or fog-free natural images and the test image. Similarly, $D_{f t}$ is the Mahalanobis distance between MVG models obtained using statistical features acquired from 500 foggy images and the test image. Lower fog density is indicated by smaller $\mathrm{D}$ values.

Each particle calculates the fitness function ' $D$ ' using equation (13). The particle can move within the search space based on its current position $x(t)$ and with a velocity $v(t)$ for each iteration ' $t$ '. Each particle is capable of storing the personal best $\mathrm{p}(\mathrm{t})$ by comparing its fitness value with the previous iteration fitness value. The personal best position of $\mathrm{i}^{\text {th }}$ particle is obtained by finding the minimum fog density ' $\mathrm{D}$ ' as follows:

$$
P_{i}(t)=\arg \min \left[D_{i}(t)\right] \quad \mathrm{i}=1,2, \ldots \mathrm{N}
$$


Also global best $g(t)$ value is obtained by comparing the best fitness value obtained by all the particles in the search space.

$$
g(t)=\arg \min \left[D\left(P_{i}(t)\right)\right]
$$

For every iteration, the personal and global best values are updated so that the optimal value is chosen when fitness function ' $\mathrm{D}$ ' becomes minimum. Also, the velocity $v(t)$ and particle position $\mathrm{x}(\mathrm{t})$ are updated using equation (16) and (17) until the iteration converges.

$$
\begin{aligned}
& v(t+1)=w v(t)+c_{1} r_{1}(p(t)-x(t))+c_{2} r_{2}(g(t)-x(t)) \\
& x(t+1)=x(t)+v(t+1)
\end{aligned}
$$

where $\mathrm{w}$ is inertia, $\mathrm{c} 1$ and $\mathrm{c} 2$ are accelerating coefficients, $\mathrm{r} 1$ and $\mathrm{r} 2$ are random values ranging between 0 and 1 . Finally an optimal global best value of $W_{d}, W_{b}, \omega$ and $t_{0}$ are used to restore the haze-free image with minimum fog density.

\section{RESUlt ANALYSIS AND DISCUSSIONS}

Qualitative and Quantitative evaluation comparing with the state-of-the-art is necessary to prove our results obtained are better, so that the visual quality of the restored image is compelling. For choosing the optimal parameters before proceeding with the proposed method we initially tested the parameters manually with different values so that the parameters vary with the fog density. After testing manually the different parameters, we finally selected weights for atmospheric estimation, aerial perspective and lower bound of transmission as the optimal parameters.

\section{A. Parameter Settings}

In the proposed method the work was carried using conventional Particle swarm optimization technique. Table I specifies the values set for different parameters like particle size, number of iterations used in our method, inertia weight and the value of accelerating coefficient and Table II specifies the lower and upper bound values of optimum variables chosen in our work. According to $\mathrm{He}$ [9], aerial perspective should not be one, so we have selected the upper bound to be 0.99. Similarly transmission lower bound should not be zero, so we have selected 0.0001 .

TABLE. I. PARAMETERS AND VALUES

\begin{tabular}{|l|l|}
\hline Parameters & Values \\
\hline Particle Size & 20 \\
\hline Iteration & 20 \\
\hline Inertia weight $(\mathrm{w})$ & 0.5 \\
\hline Accelerating coefficient $(\mathrm{c} 1, \mathrm{c} 2)$ & $\mathrm{c} 1=2$ and $\mathrm{c} 2=2$ \\
\hline
\end{tabular}

TABLE. II. LOWER AND UPPER BOUND VALUES OF OPTIMUM VARIABLES

\begin{tabular}{|l|l|l|}
\hline Optimum variables & Lower bound & Upper bound \\
\hline $\begin{array}{l}\text { Weights for atmospheric light } \\
\text { estimation }\left(\mathrm{W}_{\mathrm{d}}\right)\end{array}$ & 0 & 0.5 \\
\hline Aerial Perspective $(\omega)$ & 0 & 0.99 \\
\hline Lower bound of Transmission $(\mathrm{t} 0)$ & 0.0001 & 1 \\
\hline
\end{tabular}

\section{B. Qualitative Evaluation}

The proposed dehazing algorithm is implemented using MATLAB 2018 version and tested with the other dehazing algorithm developed by Tarel [10], He [9], Meng [21], and Sulami [22] using the source code provided by the authors. The natural outdoor hazy images are taken from the database [23], [24] to validate our performance. Fig. 5 and 7 are highlighted to show the outperformance of our method. The boxes highlighted are drawn to show that the haze-free image restored from hazy image shows detail information which wasn't visible in the input hazy image. Similarly, Fig. 6, 8 and 9 also shows the improvement in the visual quality of the image of our method. The colour and the contrast of the restored image are better than the input hazy image.

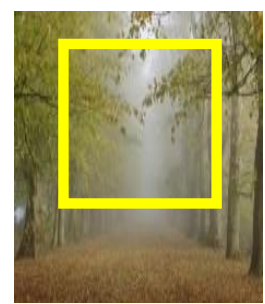

(a)



(d)

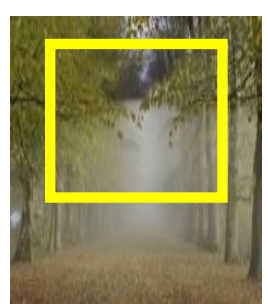

(b)

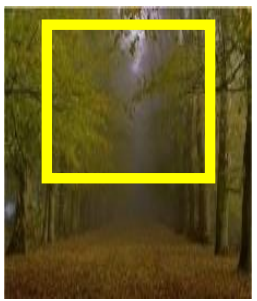

(e)

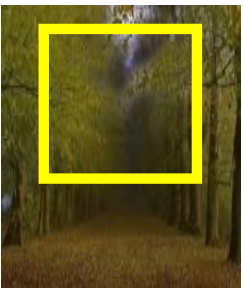

(c)

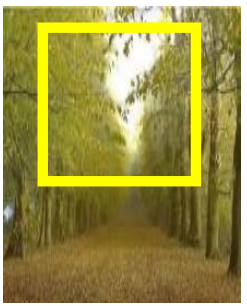

(f)
Fig. 5. Visual Comparison of (a) Forest.jpg Input Image; Dehazing Results Obtained by (b) Tarel [10]; (c) He [9]; (d) Meng [21]; (e) Sulami [22]; (f) Our Methodology.

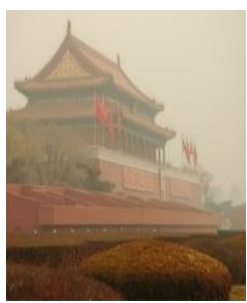

(a)

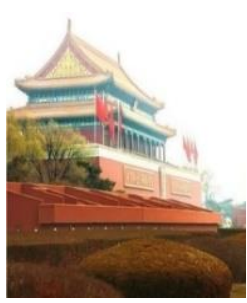

(d)

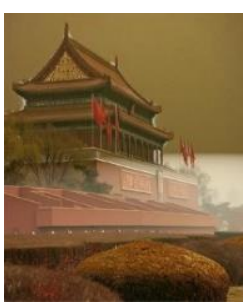

(b)

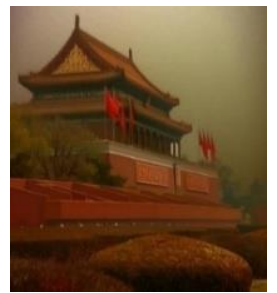

(e)



(c)



(f)
Fig. 6. Visual Comparison of (a)Tiananmen.png Input Image; Dehazing Results Obtained by (b) Tarel [10] (c) He [9]; (d) Meng [21]; (e) Sulami [22]; (f) Our Methodology. 


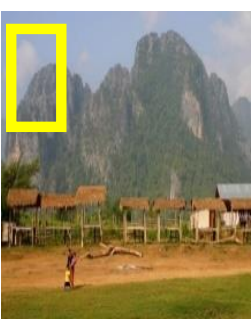

(a)

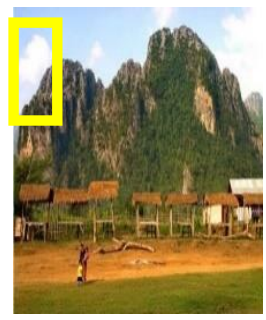

(d)

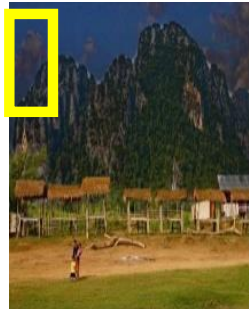

(b)

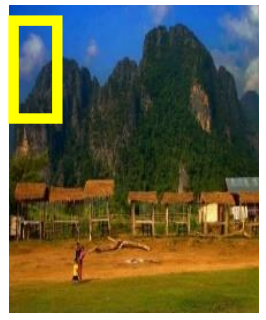

(e)

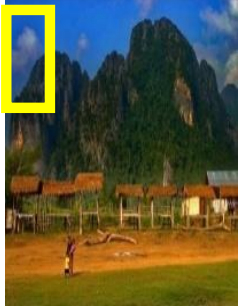

(c)

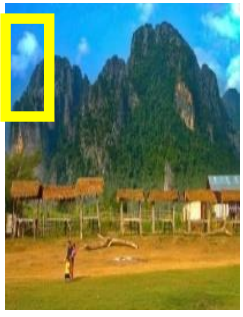

(f)
Fig. 7. Visual Comparison of (a) Mountain.png. Input Image; Dehazing Results Obtained by (b) Tarel [10] (c) He [9]; (d) Meng [21]; (e) Sulami [22] ; (f) Our Methodology.

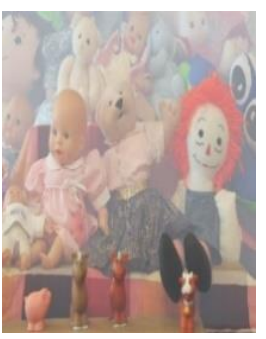

(a)

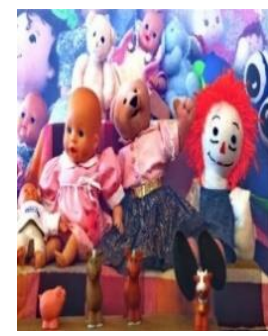

(d)



(b)



(e)

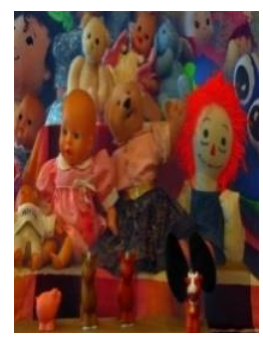

(c)



(f)
Fig. 8. Visual Comparison of (a) Toys.jpg Input Image; Dehazing Results Obtained by (b) Tarel [10] (c) He [9]; (d) Meng [21]; (e) Sulami [22]; (f) Our Methodology.

Fig. 10 shows the plot with a decrease in fog density with respect to the number of iterations applied for the input hazy image shown in Fig. 3(a). The plot clearly indicates that within three iterations the fog density almost minimizes. We tested around fifty low resolution images and found that for most of the hazy images the fog density decreases between two to ten iterations. We took 20 as the maximum iteration.



(a)

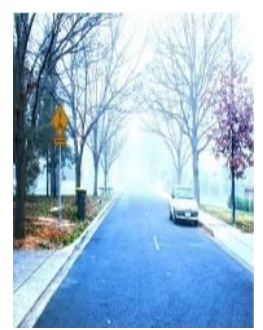

(d)

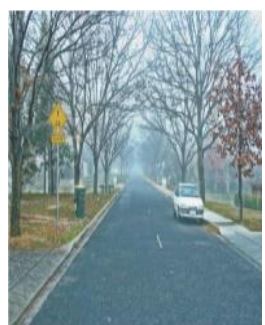

(b)

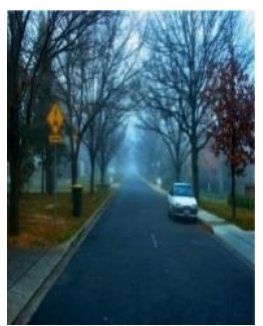

(e)

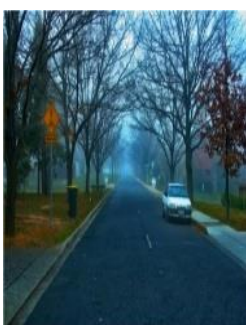

(c)

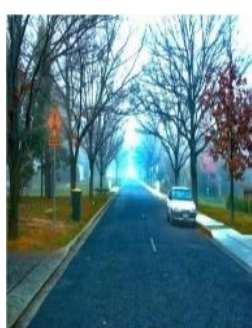

(f)
Fig. 9. Visual Comparison of (a) Sweden.jpg Input Image; Dehazing Results Obtained by (b) Tarel [10] (c) He [9]; (d) Meng [21]; (e) Sulami [22]; (f) Our Methodology.

Apart from foggy outdoor natural images, we also executed our proposed method on synthetic images obtained from Foggy Road image database (FRIDA) [24] which has images with different fog density to validate our proposed method with the state-of-the-art. Fig. 11(a) shows the fog-free synthetic images in the first column, which will be later used as ground truth images for quantitative evaluation.

Fig. 11(b) shows foggy images with different fog density added to fog-free synthetic images. The first row (second column) foggy image as a fog density of 1.5829 . Second row (second column) with a fog density of 1.1607 , the third row (second column) with a fog density of 1.5308 , fourth row (second column) with a fog density of 1.4024 and fifth row (second column) with a fog density of 1.7072 , respectively. Fig. 11(c) to Fig. 11(g) shows output restored haze-free images obtained by different dehazing technique implemented by various researchers are executed to differentiate our work on synthetic images. The hazy image will have low contrast and the colour will be faded. Our proposed method restores both colour and contrast in natural outdoor images and synthetic images. The comparison of various dehazing algorithms with our proposed method for qualitative analysis shows that our methodology is visually compelling. Since ground truth images are available in the FRIDA database we are able to perform quantitative analysis for the synthetic images by calculating Peak signal to noise ratio (PSNR) and structural similarity index (SSIM). The detail quantitative analysis or objective evaluation for synthetic images is further discussed in the next quantitative evaluation section. 


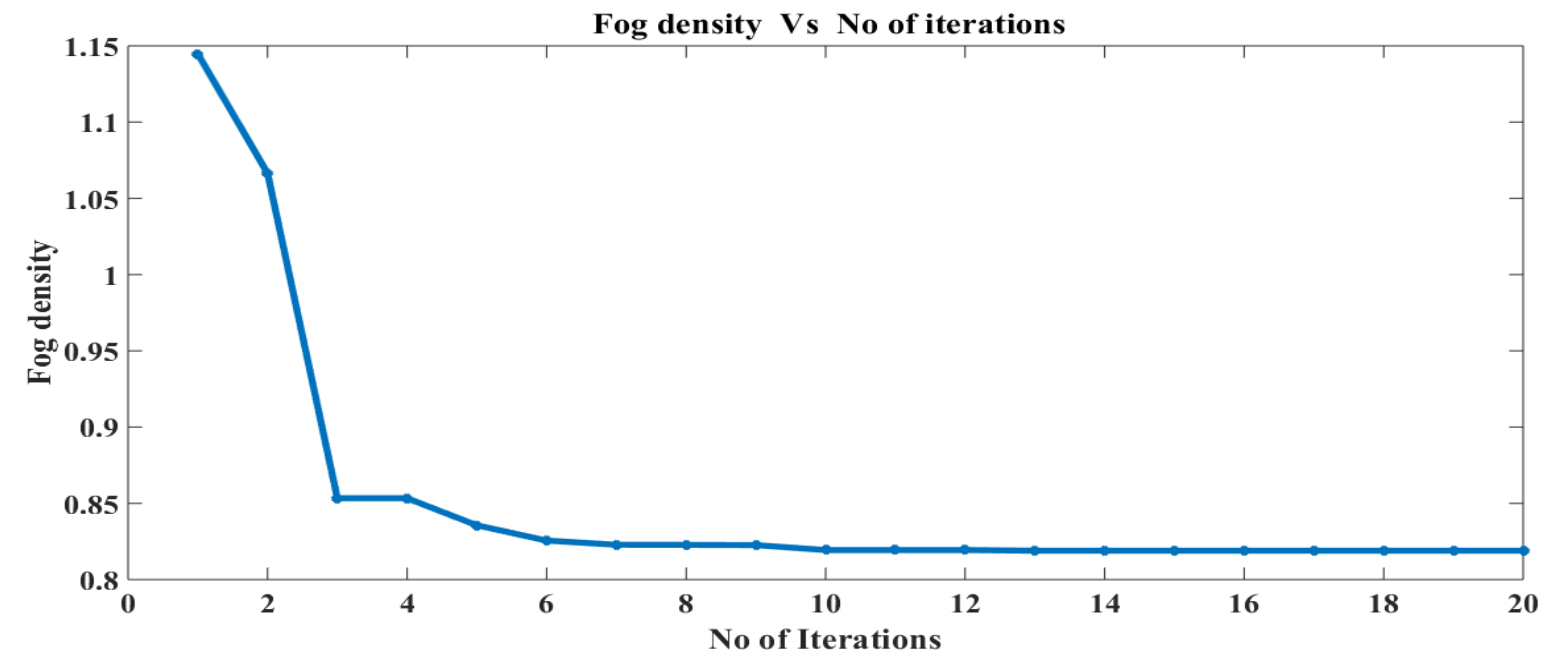

Fig. 10. Plot Indicating the Decrease in Fog Density with Respect to the Number of Iterations.


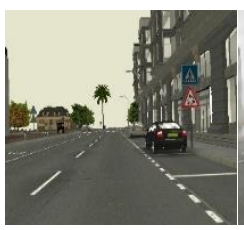

(a)

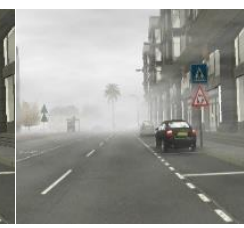

(b)

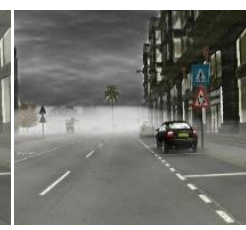

(c)

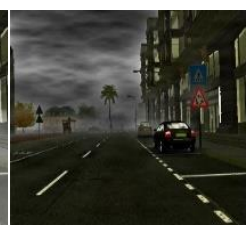

(d)

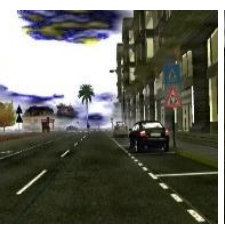

(e)

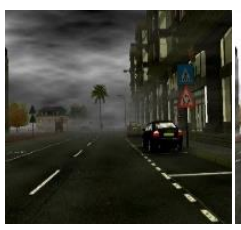

(f)

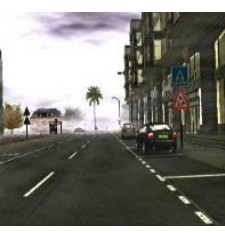

(g)

Fig. 11. Column Wise (a) Clear Synthetic Image (b) Foggy Image with different Fog Density. Results Obtained after Dehazing (c) Tarel [10] (d) He [9] (e) Meng [21] (f) Sulami [22] (g) Our Proposed Method. 


\section{Quantitative Evaluation}

For quantitative evaluation, we calculated the fog density of the input image and output restored images using equation (13) proposed by Choi [20]. Using visibility measurement of Hautiere et al. [25], we evaluated three parameters e, $\mathrm{r}$ and $\Sigma$.

The objective or quantitative evaluation of our proposed method are summarized in Tables III, IV, V and VI for the images in Fig 5, 6, 78 and 9. From Table III, we can conclude that the fog density measured from restored image using FADE evaluator tool shows that the average fog density is comparatively less than the average fog density obtained by various dehazing techniques.

Similarly Tables IV and $\mathrm{V}$ shows that the average rate of visible edges ' $e$ ' and average ratio of mean gradient ' $r_{g}$ ' obtained are comparatively higher than the values obtained from various techniques implemented by researchers who worked in this field to remove haze from images. The saturated pixels from Table VI shows almost zero for all the figures indicating that there are no saturated pixels in restored image.

TABLE. III. Fog Density EvaluATED USING FADE

\begin{tabular}{|l|l|l|l|l|l|l|}
\hline \multirow{2}{*}{ Images } & \multicolumn{6}{|l}{ Fog density (D) } \\
\cline { 2 - 7 } & $\begin{array}{l}\text { Input } \\
\text { image }\end{array}$ & $\begin{array}{l}\text { Tarel } \\
{[\mathbf{1 0}]}\end{array}$ & $\begin{array}{l}\text { He } \\
{[\mathbf{9}]}\end{array}$ & $\begin{array}{l}\text { Meng } \\
{[\mathbf{2 1}]}\end{array}$ & $\begin{array}{l}\text { Sulami } \\
{[\mathbf{2 2}]}\end{array}$ & $\begin{array}{l}\text { Proposed } \\
\text { Method }\end{array}$ \\
\hline Fig 5 & 0.389 & 0.365 & 0.190 & 0.182 & 0.222 & 0.203 \\
\hline Fig 6 & 1.345 & 0.529 & 0.383 & 0.497 & 0.434 & 0.369 \\
\hline Fig 7 & 0.583 & 0.242 & 0.232 & 0.328 & 0.242 & 0.218 \\
\hline Fig 8 & 1.229 & 0.398 & 0.286 & 0.338 & 0.258 & 0.253 \\
\hline Fig 9 & 0.963 & 0.378 & 0.215 & 0.473 & 0.224 & 0.220 \\
\hline Avg & $\mathbf{0 . 9 0 2}$ & $\mathbf{0 . 3 8 2}$ & $\mathbf{0 . 2 6 1}$ & $\mathbf{0 . 3 6 4}$ & $\mathbf{0 . 2 7 6}$ & $\mathbf{0 . 2 5 2}$ \\
\hline
\end{tabular}

TABLE. IV. RATE OF VISIBLE EDGES

\begin{tabular}{|l|l|l|l|l|l|}
\hline \multirow{2}{*}{ Images } & \multicolumn{5}{|l|}{ Rate of visible edges 'e' } \\
\cline { 2 - 6 } & Tarel [10] & He [9] & Meng [21] & $\begin{array}{l}\text { Sulami } \\
{[22]}\end{array}$ & $\begin{array}{l}\text { Proposed } \\
\text { Method }\end{array}$ \\
\hline Fig 5 & 0.10895 & 0.32917 & 0.27279 & 0.2541 & 0.3504 \\
\hline Fig 6 & 0.38368 & 0.4532 & 0.47356 & 0.47356 & 0.5246 \\
\hline Fig 7 & 0.16431 & 0.15462 & 0.0911 & 0.11851 & 0.0542 \\
\hline Fig 8 & 2.0598 & 1.9668 & 1.9097 & 2.15886 & 2.1664 \\
\hline Fig 9 & 0.52933 & 0.58948 & 0.26659 & 0.5638 & 0.6429 \\
\hline Avg & $\mathbf{0 . 6 4 9 2 1}$ & $\mathbf{0 . 6 9 8 6 5 4}$ & $\mathbf{0 . 6 0 2 7 4 8}$ & $\mathbf{0 . 7 1 3 7 6 6}$ & $\mathbf{0 . 7 4 7 7}$ \\
\hline
\end{tabular}

TABLE. V. RATIO OF MEAN GRADIENT

\begin{tabular}{|l|l|l|l|l|l|}
\hline \multirow{2}{*}{ Images } & \multicolumn{5}{|l|}{ Ratio of mean gradient ' $\mathbf{g}$ ' } \\
\cline { 2 - 6 } & Tarel [10] & $\begin{array}{l}\mathbf{H e} \\
{[\mathbf{9}]}\end{array}$ & Meng [21] & $\begin{array}{l}\text { Sulami } \\
{[\mathbf{2 2}]}\end{array}$ & $\begin{array}{l}\text { Proposed } \\
\text { Method }\end{array}$ \\
\hline Fig 5 & 2.1643 & 1.6439 & 2.3099 & 1.7616 & 2.5230 \\
\hline Fig 6 & 4.1775 & 3.2471 & 4.9593 & 3.2175 & 5.5012 \\
\hline Fig 7 & 1.3866 & 1.1098 & 2.6645 & 1.7922 & 2.1715 \\
\hline Fig 8 & 3.1120 & 1.6189 & 2.6765 & 1.7722 & 3.2172 \\
\hline Fig 9 & 2.3188 & 1.6494 & 2.1667 & 1.4151 & 2.4710 \\
\hline Avg & $\mathbf{2 . 6 3 1 8 4}$ & $\mathbf{1 . 8 5 3 8 2}$ & $\mathbf{2 . 9 5 5 3 8}$ & $\mathbf{1 . 9 9 1 7 2}$ & $\mathbf{3 . 1 7 6 7 8}$ \\
\hline
\end{tabular}

TABLE. VI. PerCentage OF SATURATEd PiXels ' $\Sigma$ '

\begin{tabular}{|l|l|l|l|l|l|}
\hline \multirow{2}{*}{ Images } & \multicolumn{5}{|l|}{ Percentage of saturated pixels ' $\boldsymbol{\Sigma}$ ' } \\
\cline { 2 - 6 } & Tarel [10] & $\begin{array}{l}\text { He } \\
{[9]}\end{array}$ & $\begin{array}{l}\text { Meng } \\
{[\mathbf{2 1}]}\end{array}$ & Sulami [22] & $\begin{array}{l}\text { Proposed } \\
\text { Method }\end{array}$ \\
\hline Fig 5 & 0.00 & 0.00 & 0.00 & 0.00 & 0.00 \\
\hline Fig 6 & 0.00 & 0.00 & 0.00 & 0.00 & 0.00 \\
\hline Fig 7 & 0.00 & 0.00 & 0.0005 & 0.00 & 0.00 \\
\hline Fig 8 & 0.00 & 0.00 & 0.00 & 0.0047 & 0.00 \\
\hline Fig 9 & 0.00 & 0.00041 & 0.00 & 0.0051 & 0.00 \\
\hline
\end{tabular}

From the results shown in Tables III, IV, V, and VI, it is evident that decrease in fog density, as well as more edges obtained in the restored image, shows the quality of the image is better than the already existing methods. Also, the number of saturated pixels are almost zero which indicates that our method is better when compared with enhancement based methods. In the sky region, Meng's and He's outcomes poor image quality and Tarel's outcomes show very clear halos around the edges.

By using the synthetic images from FRIDA database, performance analysis of the different methods have been compared with the proposed method. The two evaluation metrics measured are PSNR and SSIM. The two objective evaluation results are summarized in Tables VII and VIII. From Tables VII and VIII we can confirm that the average PSNR and SSIM are comparatively better in our proposed method for synthetic images.

Comparing the input and the output image, as per our observation our methodology shows a better quality of restored image extracting the information properly at the end of dehazing using a single color images.

TABLE. VII. PSNR OBTAINED FROM SYNTHETIC IMAGES

\begin{tabular}{|l|l|l|l|l|l|}
\hline \multirow{2}{*}{$\begin{array}{l}\text { Images } \\
\text { from }\end{array}$} & \multicolumn{5}{|l|}{ PSNR (Peak signal to noise ratio) in dB } \\
\cline { 2 - 6 } Fig 11 & Tarel [10] & $\begin{array}{l}\text { He } \\
{[9]}\end{array}$ & $\begin{array}{l}\text { Meng } \\
{[21]}\end{array}$ & $\begin{array}{l}\text { Sulami } \\
{[22]}\end{array}$ & $\begin{array}{l}\text { Proposed } \\
\text { Method }\end{array}$ \\
\hline Row 1 & 9.620 & 11.526 & 10.778 & 10.065 & 15.137 \\
\hline Row 2 & 10.276 & 10.280 & 10.423 & 10.708 & 13.096 \\
\hline Row 3 & 9.961 & 11.146 & 12.220 & 10.098 & 13.882 \\
\hline Row 4 & 10.17 & 10.495 & 12.005 & 10.816 & 12.393 \\
\hline Row 5 & 10.17 & 10.949 & 12.454 & 9.603 & 16.021 \\
\hline Avg & $\mathbf{1 0 . 0 4 1}$ & $\mathbf{1 0 . 8 7 9}$ & $\mathbf{1 1 . 5 7 6}$ & $\mathbf{1 0 . 2 5 8}$ & $\mathbf{1 4 . 1 0 6}$ \\
\hline
\end{tabular}

TABLE. VIII. SSIM OBTAINED FROM SYNTHETIC IMAGES

\begin{tabular}{|l|l|l|l|l|l|}
\hline \multirow{2}{*}{$\begin{array}{l}\text { Images } \\
\text { from }\end{array}$} & \multicolumn{5}{|l}{ SSIM (Structural Similarity Index Metric) } \\
\cline { 2 - 6 } Fig 1 1 & Tarel [10] & $\begin{array}{l}\text { He } \\
{[9]}\end{array}$ & $\begin{array}{l}\text { Meng } \\
{[21]}\end{array}$ & $\begin{array}{l}\text { Sulami } \\
{[22]}\end{array}$ & $\begin{array}{l}\text { Proposed } \\
\text { Method }\end{array}$ \\
\hline Row 1 & 0.345 & 0.419 & 0.322 & 0.453 & 0.638 \\
\hline Row 2 & 0.429 & 0.416 & 0.337 & 0.425 & 0.488 \\
\hline Row 3 & 0.469 & 0.384 & 0.440 & 0.356 & 0.486 \\
\hline Row 4 & 0.394 & 0.369 & 0.364 & 0.491 & 0.630 \\
\hline Row 5 & 0.624 & 0.371 & 0.415 & 0.36 & 0.614 \\
\hline Avg & $\mathbf{0 . 4 5 2}$ & $\mathbf{0 . 3 9 2}$ & $\mathbf{0 . 3 7 6}$ & $\mathbf{0 . 4 1 7}$ & $\mathbf{0 . 5 7 1}$ \\
\hline
\end{tabular}




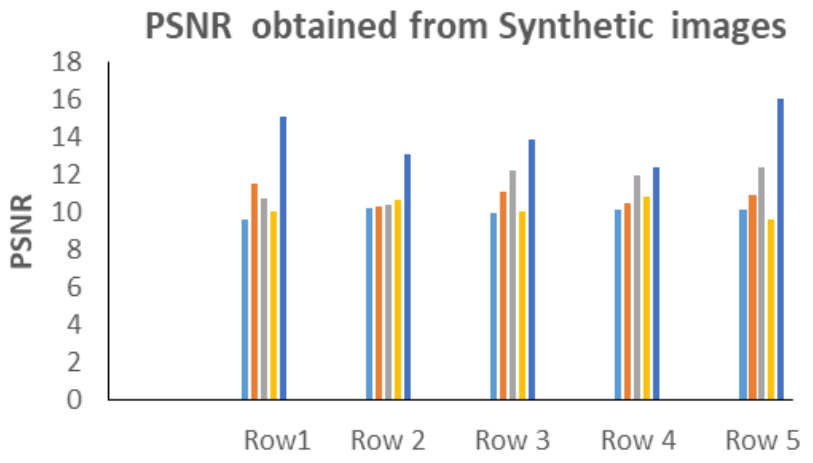

Synthetic images from Fig 11 with different fog density

Tarel $\square$ He $\square$ Meng $\square$ Sulami $\square$ Proposed method

Fig. 12. PSNR Obtained from Synthetic Images.

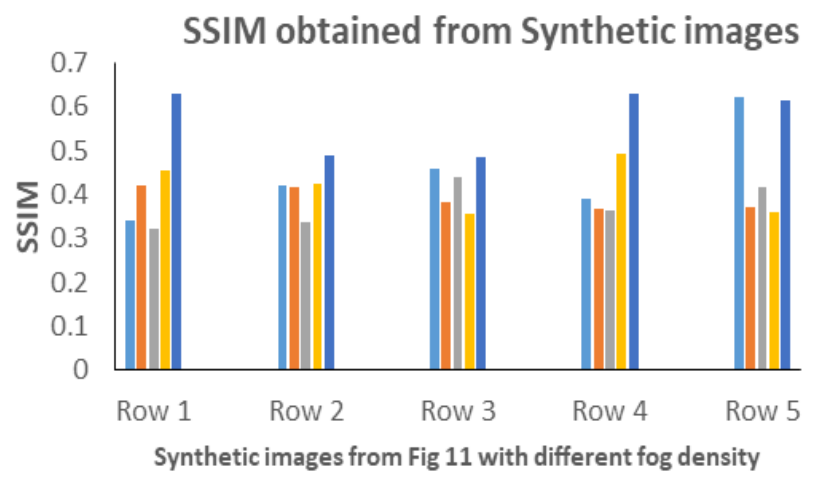

Tarel $\square$ He $\square$ Meng $\square$ Sulami $\square$ Proposed method

Fig. 13. SSIM Obtained from Synthetic Images.

The plots shown in Fig. 12 and 13 will give a clear interpretation of the results obtained by comparing the PSNR and SSIM of our proposed method with different methods.

\section{CONCLUSION}

This research demonstrates the atmospheric light estimation using Particle Swarm Optimization technique for restoring the haze-free image. This technique can exhaustively allow the search of a specific optimized value that can be used for effectively improving the visibility of the hazy regions. By choosing optimal weights for estimating the atmospheric light, haze can be removed from images more effectively. Also, the proposed method does not depend on salient objects in an image like sky regions to estimate the atmospheric light to remove haze thereby producing a perceptual quality image.

The proposed method showed variation in the results and the resultant image quality was perfectly suitable for video surveillance applications. PSNR, SSIM, rate of visible edges and restoration quality have been analyzed and the results have been compared with different methods. Since PSO is an iterative based method, the computational time is more and hence it is not suitable for applications like autonomous navigation. In future, the computational time can be reduced by implementing this technique in hardware like Field Programmable Gate Array (FPGA). However the proposed method works best for removing haze when compared to dense foggy images. Further there is a scope to develop defogging algorithms that can remove fog from images. Overall, the results obtained from the proposed method using PSO shows better results qualitatively and quantitatively.

\section{REFERENCES}

[1] Nayar, S. K., \& Narasimhan, S. G. "Vision in bad weather". In Proceedings of the Seventh IEEE International Conference on Computer Vision (Vol. 2, pp. 820-827). IEEE.1999.

[2] Narasimhan, S. G., \& Nayar, S. K. "Contrast restoration of weather degraded images". IEEE Transactions on Pattern Analysis \& Machine Intelligence, (6), 713-724, 2003.

[3] Kopf, J., Neubert, B., Chen, B., Cohen, M., Cohen-Or, D., Deussen, O., \& Lischinski, D. "Deep photo: Model-based photograph enhancement and viewing" (Vol. 27, No. 5, p. 116). ACM.,2008.

[4] Oakley, J. P., \& Bu, H. "Correction of simple contrast loss in color images". IEEE Transactions on Image Processing, 16(2), 511-522, 2007.

[5] Tripathi, A. K., \& Mukhopadhyay, S. "Removal of fog from images": A review. IETE Technical Review, 29(2), 148-156,2012.

[6] Kim, D., Jeon, C., Kang, B., \& Ko, H. "Enhancement of image degraded by fog using cost function based on human visual model". In 2008 IEEE International Conference on Multisensor Fusion and Integration for Intelligent Systems (pp. 64-67). IEEE.2008.

[7] Tan, R. T. “Visibility in bad weather from a single image”. In 2008 IEEE Conference on Computer Vision and Pattern Recognition (pp. 18). IEEE, 2008.

[8] Fattal, R. "Single image dehazing". ACM transactions on graphics (TOG), 27(3), 72, 2008.

[9] He, K., Sun, J., \& Tang, X. "Single image haze removal using dark channel prior". IEEE transactions on pattern analysis and machine intelligence, 33(12), 2341-2353, 2011.

[10] Tarel, J. P., \& Hautiere, N. "Fast visibility restoration from a single color or gray level image". In 2009 IEEE 12th International Conference on Computer Vision (pp. 2201-2208). IEEE,2009.

[11] Zhu, Qingsong, Jiaming Mai, and Ling Shao. "A fast single image haze removal algorithm using color attenuation prior." IEEE transactions on image processing 24.11 3522-3533,2015.

[12] Wang, Wencheng, et al. "Dehazing for images with large sky region." Neurocomputing 238: 365-376,2017.

[13] Li, Yunan, et al. "Single image haze removal based on haze physical characteristics and adaptive sky region detection." Neurocomputing 182: 221-234,2016.

[14] Guo, Fan, Hui Peng, and Jin Tang. "Genetic algorithm-based parameter selection approach to single image defogging." Information Processing Letters 116.10: 595-602, 2016.

[15] Zhang, Wenbo, and Xiaorong Hou. "Estimation algorithm of atmospheric light based on ant colony optimization." Proceedings of the 2017 International Conference on Intelligent Systems, Metaheuristics \& Swarm Intelligence. ACM,2017.

[16] Singh, Dilbag, and Vijay Kumar. "A Comprehensive Review of Computational Dehazing Techniques." Archives of Computational Methods in Engineering 1-19.2018.

[17] Eberhart, Russell, and James Kennedy. "A new optimizer using particle swarm theory." MHS'95. Proceedings of the Sixth International Symposium on Micro Machine and Human Science. IEEE, 1995.

[18] Shiau, Y. H., Chen, P. Y., Yang, H. Y., Chen, C. H., \& Wang, S. S. "Weighted haze removal method with halo prevention". Journal of Visual Communication and Image Representation, 25(2), 445-453, 2014.

[19] He, Kaiming, Jian Sun, and Xiaoou Tang. "Guided image filtering." European conference on computer vision. Springer, Berlin, Heidelberg, 2010. 
[20] Choi, L. K., You, J., \& Bovik, A. C. "Referenceless prediction of perceptual fog density and perceptual image defogging". IEEE Transactions on Image Processing, 24(11), 3888-3901, 2015.

[21] Meng, G., Wang, Y., Duan, J., Xiang, S., \& Pan, C. "Efficient image dehazing with boundary constraint and contextual regularization". In Proceedings of the IEEE international conference on computer vision (pp. 617-624), 2013.

[22] Sulami, M., Glatzer, I., Fattal, R., \& Werman, M. "Automatic recovery of the atmospheric light in hazy images". In 2014 IEEE International Conference on Computational Photography (ICCP) (pp. 1-11). IEEE,2014.
[23] Ma, Kede, Wentao Liu, and Zhou Wang. "Perceptual evaluation of single image dehazing algorithms." 2015 IEEE International Conference on Image Processing (ICIP). IEEE, 2015.

[24] Tarel, Jean-Philippe, et al. "Improved visibility of road scene images under heterogeneous fog." 2010 IEEE Intelligent Vehicles Symposium. IEEE, 2010.

[25] Hautiere, Nicolas, et al. "Blind contrast enhancement assessment by gradient ratioing at visible edges." Image Analysis \& Stereology 27.2: 87-95, 2008. 